\title{
Which-way Information in a Nested Mach-Zehnder Interferometer
}

\author{
Václav Potoček* \\ School of Physics and Astronomy, SUPA, University of Glasgow, Glasgow G12 8QQ, UK and \\ Czech Technical University in Prague, FNSPE, Břehová 7, 11519 Praha 1, Czech Republic \\ Gergely Ferenczi* \\ School of Physics and Astronomy, SUPA, University of Glasgow, Glasgow G12 8QQ, UK
}

\begin{abstract}
Recently Danan et al. [Phys. Rev. Lett. 111, 240402 (2013)] have performed a much discussed experiment in which which-way information was obtained from the light in a nested Mach-Zehnder interferometer by weak measurement. The presented analysis using the two state vector formalism drew the conclusions that the photons followed disconnected paths. We analyse this experiment using standard quantum optical methods and arrive at analytical expressions that match the experimental results without the need for such disconnected photon paths. We also propose a simple amendment to the experiment capable of displaying new phenomena highlighting the advantages of our description.
\end{abstract}

PACS numbers: 42.15.Dp, 42.25.-p, 42.25.Hz

\section{INTRODUCTION}

Recently there has been a proposal by Vaidman [1] to obtain which-way information from a photon in a nested Mach-Zehnder interferometer (MZI) by weak measurement. This has been implemented by Danan et al. [2] where the measurement was performed by weakly marking each path by reflecting the light off of a vibrating mirror (see Fig. 1) and measuring the time varying intensity difference across two halves of a quad-cell detector placed at one of the outputs. This has generated much interest in both theoretical $[3,4]$ and experimental [5-11] contexts due mainly to the controversial conclusion that the photons follow disconnected paths in the interferometer.

The point of issue arises when maximum possible destructive interference towards mirror $F$ is arranged. The assumption that this is complete destructive interference, i.e., that light reaching the detector $D$ could have gone only via the lower path in Fig. 1, led to the curious observation of the vibrational frequencies of mirrors $A$ and $B$ along with that of $C$ in the spectrum of the signal and yet the absence of frequencies of $E$ and $F$. A further surprise - that at first seems to support the assumption of complete destructive interference in the inner interferometer - was the disappearance of all three frequencies $A, B$ and $C$ upon blocking the lower path containing only mirror $C$, suggesting an interpretation that the photons did not reach the mirrors $A$ and $B$ via $E$ and $F$ but rather as a disconnected part of their path via $C$. The explanation put forward by Danan et al. is based on the two-state vector formalism (TSVF) pioneered by Aharonov et al. [12], linking the presence (or absence) of the peaks at $A, B(E, F)$ to the simultaneous presence (or the lack of) both forward- and backward-propagating states from the source and the detector.

\footnotetext{
* Both authors contributed equally to this work.

Send correspondence to: vaclav.potocek@glasgow.ac.uk
}

Once one acknowledges that there is a non-negligible leakage from the inner MZI due to the fact that the two paths are partially distinguished, i.e., that light from paths $A$ and $B$ does have a means of reaching the detector, the mystery vanishes. Salih [5] and Saldanha [6] both recognised the significance of the leakage. Salih argued that in the case that true complete destructive interference towards mirror $F$ is arranged by marking paths $A$ and $B$ with degenerate frequencies with the mirrors vibrating with opposite phase no ground is given for the claims of Danan et al. Saldanha has provided a phenomenological description of the interference by showing numerically that the leakage acts as a first-order correction to the beam profile and that this leakage, when mixing with the light from arm $C$, acts to displace the light beam in the transverse plane, thereby imprinting it with the signatures of mirrors $A$ and $B$. Moreover, Danan et al., in the supplementary material appended to their paper [13], have themselves shown that there is a non-negligible leakage by blocking light from mirror $F$ and observing the peaks corresponding to mirrors $A$ and $B$ disappear, although this was interpreted by them as evidence for the lack of electronic noise in the set-up. Bartkiewicz et al. [11] have attempted to provide a minimal fully quantum treatment working in the frequency domain only. However they have assumed that the effect of the vibrating mirrors is to imprint the which-way information using mutually orthogonal (i.e. perfectly distinguishable) states, hence their assumptions as well as results disagree with those of the experiment they are describing.

We put Saldanha's description on a simple analytical footing by modelling the effect of the mirrors as a firstorder Hermite-Gaussian perturbation to the light in the interferometer. We derive an effective observable that shows explicitly that the perturbed portion of the light (carrying the which-way information) must mix with the unperturbed part to give a non-vanishing signal. Within this context we discuss the experimental results presented 


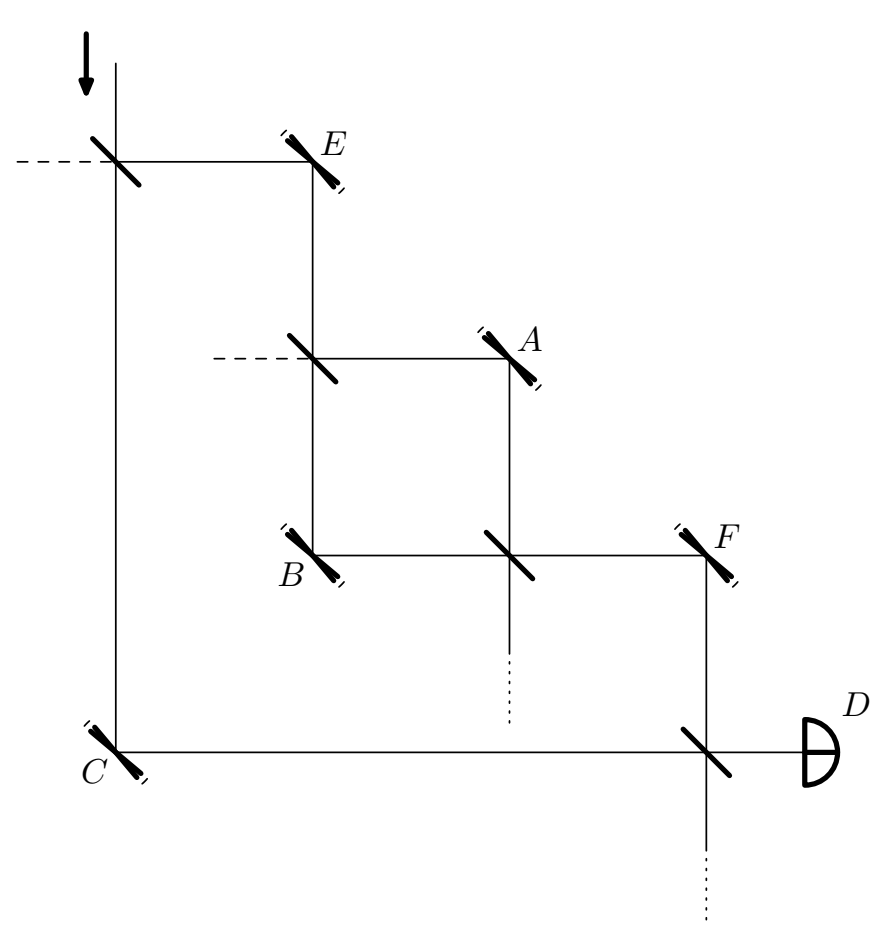

FIG. 1. The schematic of the experiment. The symbols labelled $A, B, C, E, F$ denote the vibrating mirrors and $D$ denotes a two-cell detector. The two dashed lines denote blind input arms of beam splitters and the two dotted lines output arms which are not measured in the experiment.

in [2]. Once the formalism is properly set up simple arguments based on the trade-off between path distinguishability and visibility of interference can be employed to understand the results in all cases, in particular in the counter intuitive case of arm $C$ being blocked for which a simple analysis in the TSVF fails [7]. Further we show that by appropriate tuning of the path lengths the signatures of certain mirrors may be hidden. We work throughout in the standard quantum optical formalism. The treatment may be transposed to the classical domain by using a coherent state as an input or by replacing annihilation and creation operators by complex field amplitudes. The validity of our quantum treatment extends to fermion interferometry such as that based on neutrons, as all the relevant states and observables can also be constructed for fermions.

Our work is structured as follows: In Section II we begin by establishing the formalism to be used to describe the interferometer. Despite drawing grounds for the employed simplifications from the experimental setup [2], we emphasize that this is not a part of the description of the interferometer itself; experienced readers may review only the key points and skip ahead to the following section. In Section III, we use the formalism in a quantum path sum approach to obtain an analytical description of the detector output and discuss the immediate observations made possible by our method. In Section IV, we

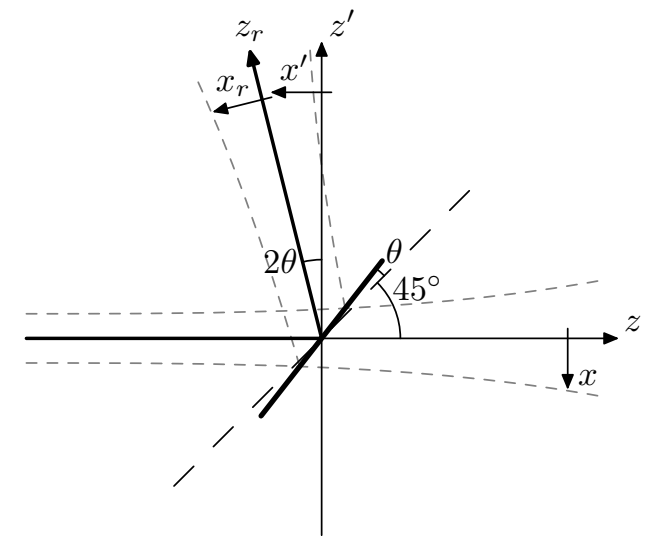

FIG. 2. A tilted mirror and the original and reflected coordinate systems. The system $\left(x_{r}, y_{r}, z_{r}\right)$ corresponds to $(x, y, z)$ reflected with respect to the actual location of the mirror while $\left(x^{\prime}, y^{\prime}, z^{\prime}\right)$ is reflected with respect to $45^{\circ}$. The $y$ axis is not affected by the reflection $\left(y=y^{\prime}=y_{r}\right)$ and is not plotted. Both the tilt angle $\theta$ and the beam divergence are vastly exaggerated.

match our results to those measured in [2]. In Section V, we propose a small amendment of the system in which novel phenomena can be observed. Finally, we conclude our results.

\section{PRODUCTION AND DETECTION OF SLIGHTLY TILTED GAUSSIAN BEAMS}

Let us consider a monochromatic paraxial beam going along the $z$ axis incident at a mirror inclined at an angle $\pi / 4+\theta$ to its axis in the $x z$ plane, as illustrated in Fig. 2 . Inspired by the experiment [2], we will only consider minuscule tilt angles $\theta$ for which no point of the mirror's surface is displaced more than a fraction of a wavelength over the principal cross section of the beam, as modelled by its beam waist $w_{0}$. Mathematically, we will assume the condition

$$
w_{0} \theta \ll \lambda,
$$

or

$$
k w_{0} \theta \ll 2 \pi,
$$

where $\lambda$ is the wavelength and $k=2 \pi / \lambda$ the wave number. For the typical values in the experiment, $\lambda \approx$ $700 \mathrm{~nm}, w_{0} \approx 1 \mathrm{~mm}$, and $\theta_{\max } \approx 300 \mathrm{nrad}$, the peak value of the left hand side of (1) is three orders of magnitude smaller than $\pi$. In an optical table setting the $s$ and $p$-polarizations are maintained throughout the setup, so for an unpolarized light or a fixed input polarization a scalar wave description is fully sufficient. Moreover, the reflected beam is simply an analytic continuation of the incident beam in the reflected coordinate system $\left(x_{r}, y_{r}, z_{r}\right)$ as per Fig. 2. 
It will be more practical to work in the untilted coordinate system $\left(x^{\prime}, y^{\prime}, z^{\prime}\right)$ where the reflected beam is rotated by the angle $2 \theta$. If the incident beam is paraxial with respect to $z$ and described by a scalar complex field $u(x, y, z, t)=\psi(x, y, z) e^{i k z-i \omega t}$, the reflected beam is paraxial with respect to $z^{\prime}$ and similarly described by

$\psi^{\prime}\left(x^{\prime}, y^{\prime}, z^{\prime}\right)=e^{2 i k \theta x^{\prime}-2 i k \theta^{2}\left(z^{\prime}-z_{M}^{\prime}\right)} \psi\left(x^{\prime}-2 \theta\left(z^{\prime}-z_{M}^{\prime}\right), y^{\prime}, z^{\prime}\right)$,

where $z_{M}^{\prime}$ denotes the $z$ coordinate of the point of intersection of the mirror with the beam axis. This transformation represents a symmetry of the paraxial wave equation,

$$
2 i k \frac{\partial \psi(x, y, z)}{\partial z}=-\left(\frac{\partial^{2}}{\partial x^{2}}+\frac{\partial^{2}}{\partial y^{2}}\right) \psi(x, y, z)
$$

with respect to shear transforms [14]. At $z^{\prime}=z_{M}^{\prime}$, and with the condition (1) in mind, (2) can be written as

$$
\begin{aligned}
\psi^{\prime}\left(x^{\prime}, y^{\prime}, z_{M}^{\prime}\right)= & \psi\left(x^{\prime}, y^{\prime}, z_{M}^{\prime}\right)+2 i k \theta x^{\prime} \psi\left(x^{\prime}, y^{\prime}, z_{M}^{\prime}\right) \\
& +O\left(\left(k w_{0} \theta\right)^{2}\right) .
\end{aligned}
$$

Specifically, if the incident wave is Gaussian of the form

$$
\psi(x, y, z)=\psi_{00}(x, y, z)=\sqrt{\frac{2}{\pi w_{0}^{2}}} \frac{z_{R}}{z_{R}+i z} e^{-\frac{k\left(x^{2}+y^{2}\right)}{2\left(z_{R}+i z\right)}},
$$

where $w_{0}$ is the beam waist and $z_{R}=\frac{1}{2} k w_{0}^{2}$ the Rayleigh range (a distance from the beam waist where the width expands by a factor of $\sqrt{2}$ ), the second term of the right hand side of (4) represents a first-order HermiteGaussian wavefront superimposed on the former profile. Indeed, the corresponding $\mathrm{TEM}_{10}$ wave is described by

$$
\begin{aligned}
\psi_{10}(x, y, z) & =\sqrt{\frac{2}{\pi w_{0}^{2}}}\left(\frac{z_{R}}{z_{R}+i z}\right)^{2} \frac{2 x}{w_{0}} e^{-\frac{k\left(x^{2}+y^{2}\right)}{2\left(z_{R}+i z\right)}} \\
& =\frac{2 x}{w_{0}} \frac{z_{R}}{z_{R}+i z} \psi_{00}(x, y, z),
\end{aligned}
$$

so after the reflection (4), it holds that

$$
\begin{aligned}
\psi^{\prime}\left(x^{\prime}, y^{\prime}, z^{\prime}\right)= & \psi_{00}\left(x^{\prime}, y^{\prime}, z^{\prime}\right)+i k w_{0} \theta \frac{z_{R}+i z_{M}}{z_{R}} \psi_{10}\left(x^{\prime}, y^{\prime}, z^{\prime}\right) \\
& +O\left(\left(k w_{0} \theta\right)^{2}\right) .
\end{aligned}
$$

Note that except for the immediate neighbourhood of the mirror we don't need to distinguish between $(x, y, z)$ and $\left(x^{\prime}, y^{\prime}, z^{\prime}\right)$ : for the incident (reflected) part of the beam, the original (primed) coordinate system is implicitly used. The change occurs at $z=z^{\prime}=z_{M}$.

The above results are valid for a monochromatic case with an error of $O\left(\left(w_{0} / \lambda\right)^{-4}\right)$ due to the paraxial wave approximation used. Under a narrow bandwidth assumption, their validity can be extended to quasimonochromatic waves, affecting only the propagation factor $e^{i k z-i \omega t}$. The resulting error terms are of the order $O\left(\left(\Delta \omega / \omega_{0}\right)\left(w_{0} / \lambda\right)^{-2}\right)$ and for a typical optical laser source of coherence length $\sim 1 \mathrm{~m}$ are strongly dominated by the former. In other words, we can assume that for a sufficiently narrow vicinity of $\omega_{0}$ the spatial mode structure does not change significantly. This allows us to define annihilation and creation operators indexed by the $m, n$ indices of TEM waves and frequency, satisfying

$$
\left[\hat{a}_{m n}(\omega), \hat{a}_{m^{\prime} n^{\prime}}^{\dagger}\left(\omega^{\prime}\right)\right]=\delta_{m m^{\prime}} \delta_{n n^{\prime}} \delta\left(\omega-\omega^{\prime}\right),
$$

such that the field operator in the scalar description and under the two approximations taken becomes

$$
\begin{aligned}
\hat{A}(x, y, z, t)= & \sqrt{\frac{\hbar}{4 \pi \epsilon_{0} \omega_{0} c}} \sum_{m, n=0}^{+\infty} \psi_{m n}(x, y, z) \times \\
& \int_{0}^{+\infty} \mathrm{d} \omega e^{-i \omega(t-z / c)} \hat{a}_{m n}(\omega)+\text { h.c. }
\end{aligned}
$$

Following the construction in [15], we further define

$$
\hat{a}_{m n}(t):=\frac{1}{\sqrt{2 \pi}} \int_{\mathbb{R}} \mathrm{d} \omega e^{-i \omega t} \hat{a}_{m n}(\omega)
$$

and write the longitudinal component of the Poynting vector as

$$
\begin{aligned}
\hat{S}(x, y, z, t)= & \hbar \omega_{0} \sum_{m, n, m^{\prime}, n^{\prime}=0}^{+\infty} \psi_{m n}^{*}(x, y, z) \psi_{m^{\prime} n^{\prime}}(x, y, z) \times \\
& \hat{a}_{m n}^{\dagger}\left(t-\frac{z}{c}\right) \hat{a}_{m^{\prime} n^{\prime}}\left(t-\frac{z}{c}\right) .
\end{aligned}
$$

This clearly represents the energy of one photon at the central frequency (owing to the narrow bandwidth approximation used) multiplied by the flux of particles at retarded time. In the case of fermionic particles the derivation will be different but the flux can be used without modifications. For a stationary beam, the expectation value of the flux is constant in time. This is possible regardless of the type of particle.

Let us now consider a quad-cell detector placed further along the beam at $z=z_{D}$. The idealized model measures the total intensity difference between two half-planes in the direction of the displacement:

$\widehat{\Delta I}(t)=\int_{0}^{+\infty} \mathrm{d} x \int_{\mathbb{R}} \mathrm{d} y \hat{S}\left(x, y, z_{D}, t\right)-\int_{-\infty}^{0} \mathrm{~d} x \int_{\mathbb{R}} \mathrm{d} y \hat{S}\left(x, y, z_{D}, t\right)$.

If, according to (6), the modes $\mathrm{TEM}_{00}$ and $\mathrm{TEM}_{10}$ are sufficient to describe the state of the beam at any instant, we can restrict the operator to the relevant subspace to get an effective observable. Doing so and carrying out the explicit integration, this gives

$$
\widehat{\Delta I}_{e}(t)=I_{0}\left(e^{-i \zeta\left(z_{D}\right)} \hat{a}_{00}^{\dagger}\left(t^{\prime}\right) \hat{a}_{10}\left(t^{\prime}\right)+\text { h.c. }\right)
$$

where

$$
I_{0}=\hbar \omega_{0} \sqrt{\frac{2}{\pi}}, \quad t^{\prime}=t-\frac{z}{c}, \quad \text { and } \quad \zeta\left(z_{D}\right)=\arctan \frac{z_{D}}{z_{R}}
$$


is the Gouy phase. (We note that if the detector is not geometrically ideal, as assumed above, only the prefactor $I_{0}$ changes to reflect the geometry as long as it is symmetric with respect to the axis of the undisplaced beam.) From this formula, we see that it is of great importance that both the carrier Gaussian wave and the Hermite-Gaussian correction are incident at the detector plane simultaneously for a nonzero signal to be obtained. Moreover, the response is linear in both the amplitudes, which will be crucial in the following.

Let us examine the expectation value of the observable (7) for a single-particle state with the spatial structure (6). Let $f(t)$ be some $L^{2}$ function representing the temporal shape of the pulse (slowly varying, to comply with the narrow bandwidth assumption). Thus, we write

$$
|\psi\rangle=\int_{\mathbb{R}} \mathrm{d} t f(t)\left(\hat{a}_{00}^{\dagger}(t)+i k w_{0} \theta \frac{z_{R}+i z_{M}}{z_{R}} \hat{a}_{10}^{\dagger}(t)\right)|0\rangle .
$$

Then for $t^{\prime}=t-z / c$,

$$
\left\langle\psi\left|\hat{a}_{00}^{\dagger}\left(t^{\prime}\right) \hat{a}_{10}\left(t^{\prime}\right)\right| \psi\right\rangle=i k w_{0} \theta \frac{z_{R}+i z_{M}}{z_{R}}\left|f\left(t^{\prime}\right)\right|^{2}
$$

and hence

$$
\left\langle\psi\left|\widehat{\Delta I}\left(t^{\prime}\right)\right| \psi\right\rangle=\left\langle\psi\left|\widehat{\Delta I} \widehat{I}_{e}\left(t^{\prime}\right)\right| \psi\right\rangle=2 k w_{0} \theta I_{0} \frac{z_{D}-z_{M}}{\sqrt{z_{R}^{2}+z_{D}^{2}}}\left|f\left(t^{\prime}\right)\right|^{2}
$$

This result illustrates the fact that right after the mirror $\left(z_{D} \approx z_{M}\right)$, the Hermite-Gaussian component only modifies the local phase of the wave profile but the intensity remains parity-symmetric, resulting in zero $\langle\widehat{\Delta I}\rangle$. The further the detector is placed from the mirror, the longer optical length both the waves propagate freely, resulting in their superposition forming a displaced Gaussian profile as argued by Saldanha [6] and producing a nonzero differential signal. With constant $I_{0}, z_{M}$, and $z_{D}$, the differential signal is proportional to $\theta$ (within the small angle approximation). At large distances the differential intensity saturates at the limit value of $2 I_{0} k w_{0} \theta$.

In the following, we will take the liberty of leaving out the time argument and the second spatial index (which is always zero) of $\hat{a}$ for the sake of brevity, as well as the time argument of $\widehat{\Delta I}$, e.g.,

$$
\widehat{\Delta I}=I_{0}\left(e^{-i \zeta\left(z_{D}\right)} \hat{a}_{0}^{\dagger} \hat{a}_{1}+\text { h.c. }\right)
$$

The result (11) indicates that any time dependence of the input state will only serve as a prefactor (in retarded time) in the output state within the global assumptions.

One more important observation is that (11) does not significantly depend on small variations in path length (of the order of $\lambda$ ). This allows us to denote in the following the position of the detector by a single $z_{D}$ coordinate even in the case of interference of several distinct paths including small differences in optical path length to obtain relative phases.

\section{CONTRIBUTION OF THE DISTINCT PATHS}

In Fig. 1, we can identify three possible paths from the input to the detector, determined by a reflection off the mirrors $E, A, F$, or $E, B, F$, or $C$ only. We will denote the three paths by $A, B$, and $C$. The reflectivities of the four beam splitters at the diagonal of Fig. 1 are chosen such that each of these paths enters the path sum with an equal weight of $1 / 3$. This is done in [2] by means of polarizers and polarizing beam splitters but in a way that is indistinguishable from polarization-independent beam splitters with a fixed input polarization at the measurement stage. The phases of the reflection and transmission coefficients will be accounted for later.

The mirrors $A, B, C, E, F$ oscillate at frequencies $\omega_{A}$ to $\omega_{F}$, which are assumed to be all mutually different but each of them many orders of magnitude smaller than $\omega_{0}$. (In [2] the former are of the order of hundreds of $\mathrm{Hz}$, as compared to the optical frequency of $\omega_{0}$. ) In this case there is no need to take into account any timefrequency uncertainty or finite propagation time effects and the time dependence of the angles $\theta_{M}(t)$ can be simply reflected in an explicit time dependence of the optical state incident at the detector.

We build upon (9) to describe the effect of reflections off of several tilted mirrors, whose angles $\theta$ allow for slow time variation. We will consider the tilts of all the mirrors, denoted $\theta_{A}(t)$ through $\theta_{F}(t)$, to be bounded by a common $\theta$ at all times. Clearly, the Gaussian term remains after each reflection with the same amplitude (up to a correction of the order $\left.O\left[\left(k w_{0} \theta\right)^{2}\right]\right)$ and represents a "carrier wave" common to the whole optical path. Trivially, each of the mirrors adds a first-order HermiteGaussian component with an amplitude

$$
i k w_{0} \theta_{M}(t) \frac{z_{R}+i z_{M}}{z_{R}}
$$

relative to the carrier wave, where $\theta_{M}(t)$ is the tilt of the mirror $M \in\{A, B, C, E, F\}$ and $z_{M}$ represents the optical distance from the source, where the beam is collimated. (It is important to add that the rectangular configuration of the interferometer defines the ' $x$ ' direction, and thus the orientation of Hermite-Gaussian modes, in every path segment unambiguously.) In fact, summing these contributions is sufficient to describe the effect of several consecutive mirrors. Of course, each of the successive mirrors will also apply a transformation to the side terms added by the previous reflections but these will be second order in $k w_{0} \theta$ and can be neglected.

Furthermore, in [2] the mirrors are located at similar positions $z_{M} \ll z_{R}$ whereas for the detector it holds that $z_{D} \lesssim z_{R}$. In order to illustrate the core of our argument without the burden of unnecessary detail, we will reflect this in the following by leaving out terms of the form $z_{M} / z_{R}, M \in\{A, B, C, E, F\}$, so that the term $\left(z_{R}+\right.$ $\left.i z_{M}\right) / z_{R}$ in equation (13) reduces to 1 . The analysis is equally tractable without this simplification. 
In this setting we find that the path $A$ transforms an initial state $\hat{a}_{0}^{\dagger}|0\rangle$ to

$$
\left(\hat{a}_{0}^{\dagger}+i\left(\delta_{E}(t)+\delta_{A}(t)+\delta_{F}(t)\right) \hat{a}_{1}^{\dagger}\right)|0\rangle+O\left(\delta^{2}\right)
$$

where the notation

$$
\delta_{M}(t)=k w_{0} \theta_{M}(t), \quad \delta=k w_{0} \theta
$$

has been introduced. Similarly, the paths $B$ and $C$ result in contributions of the form

$$
\begin{aligned}
& \left(\hat{a}_{0}^{\dagger}+i\left(\delta_{E}(t)+\delta_{B}(t)+\delta_{F}(t)\right) \hat{a}_{1}^{\dagger}\right)|0\rangle+O\left(\delta^{2}\right), \\
& \left(\hat{a}_{0}^{\dagger}+i \delta_{C}(t) \hat{a}_{1}^{\dagger}\right)|0\rangle+O\left(\delta^{2}\right)
\end{aligned}
$$

respectively. The three above lines contribute to a path sum, after multiplication by the weight factor $1 / 3$ and relative phase factors $e^{i \phi_{A}}, e^{i \phi_{B}}$, and $e^{i \phi_{C}}$ which include path length differences as well as the phases of the reflection and transmission coefficients of the beam splitters encountered along the paths. The resulting state can be written as

$$
\left|\psi_{\text {out }}(t)\right\rangle=\frac{1}{3}\left(\alpha_{0} \hat{a}_{0}^{\dagger}+\sum_{M \in\{A, B, C, E, F\}} \alpha_{M}(t) \hat{a}_{1}^{\dagger}\right)|0\rangle+O\left(\delta^{2}\right),
$$

where

$$
\begin{aligned}
\alpha_{0} & =e^{i \phi_{A}}+e^{i \phi_{B}}+e^{i \phi_{C}}, & & \\
\alpha_{M}(t) & =e^{i \phi_{M}} i \delta_{M}(t), & & M \in\{A, B, C\}, \\
\alpha_{M}(t) & =\left(e^{i \phi_{A}}+e^{i \phi_{B}}\right) i \delta_{M}(t), & & M \in\{E, F\} .
\end{aligned}
$$

From this general form of the output state we can make several observations:

- The Gaussian term, $\hat{a}_{0}^{\dagger}|0\rangle$, has the same coefficient (to the first order) as if all the mirrors were stationary with zero tilt angles $\theta_{M}$, therefore it represents the part of the state with full visibility of interference but no distinction between the paths.

- All of the which-way information (i.e., values dependent on $\left.\theta_{M}(t)\right)$ is contained in the coefficient of the Hermite-Gaussian component $\hat{a}_{1}^{\dagger}|0\rangle$ and prevented from further influencing the carrier wave by the orthogonality of the two modes.
- The amplitude of $\hat{a}_{1}^{\dagger}|0\rangle$ is linear in all angles $\theta_{M}(t)$. The constant of proportionality will always be nonzero for $\alpha_{A}(t), \alpha_{B}(t)$, and $\alpha_{C}(t)$, unless the corresponding path is blocked. However, $\alpha_{E}(t)$ and $\alpha_{F}(t)$ in the $\hat{a}_{1}^{\dagger}|0\rangle$ component are undergoing interference of the same quality as paths $A$ and $B$ do in the $\hat{a}_{0}^{\dagger}|0\rangle$ component.

The last point is a direct consequence of the fact that the terms containing $\delta_{A}(t), \delta_{B}(t)$, and $\delta_{C}(t)$ come from exactly one of the three paths each, while the terms containing $\delta_{E}(t)$ or $\delta_{F}(t)$ appear in both paths $A$ and $B$ and therefore interfere the same way as the carrier Gaussian wave in these two paths. In other words, the former three terms represent a fully decisive which-way information, which then can not undergo further interference. The latter two terms represent a partial which-way information, which rules out path $C$ but retains the visibility of the interference of the remaining two paths.

We propose this simple explanation as a fully traditional alternative to the explanation given in the conclusions section of [2], and moreover, one to be readily foreseen from the fact that which-way marking at mirrors $E$ and $F$ does not distinguish between two interfering paths.

\section{ANALYSIS OF THE RELEVANT CASES}

Quantifying our results, direct application of (12) yields the differential signal as

$$
\left\langle\psi_{\text {out }}(t)|\widehat{\Delta I}| \psi_{\text {out }}(t)\right\rangle=\frac{2 I_{0}}{9} \sum_{M} \Re\left(e^{-i \zeta\left(z_{D}\right)} \alpha_{0}^{*} \alpha_{M}(t)\right)
$$

Inserting for the values of $\alpha_{M}$ results in meaningful simplification only in the case where the phases $\phi_{A}, \phi_{B}$, $\phi_{C}$ are equal or differ by integer multiples of $\pi$. By setting $e^{i \phi_{A}}=e^{i \phi_{C}}=e^{i \Phi}$ and $e^{i \phi_{B}}= \pm e^{i \Phi}$ in (17) one can see that $\alpha_{0}^{*} \alpha_{M}$ is purely imaginary under this restriction and $\left|\alpha_{0}\right|$ takes the value $2 \pm 1$ for the constructive $(+)$ and destructive $(-)$ interferences arranged in the inner MZI. The expectation value of the differential intensity is then

$$
\left\langle\psi_{\text {out }}(t)|\widehat{\Delta I}| \psi_{\text {out }}(t)\right\rangle=\frac{2 I_{0}}{9} \sin \zeta\left(z_{D}\right)\left|\alpha_{0}\right|\left(\delta_{A}(t) \pm \delta_{B}(t)+\delta_{C}(t)+\left(\left|\alpha_{0}\right|-1\right)\left(\delta_{E}(t)+\delta_{F}(t)\right)\right)+O\left(\delta^{2}\right)
$$

This result agrees perfectly with the experimental results in [2] in the studied cases with path $C$ opened. In particular, if all the three paths are aligned for constructive interference, then $e^{i \phi_{A}}=e^{i \phi_{B}}=e^{i \phi_{C}}$ and $\left|\alpha_{0}\right|=3$. This results in equal sensitivities to displacements from mirrors $A, B$ and $C$ and double sensitivities to displacements 


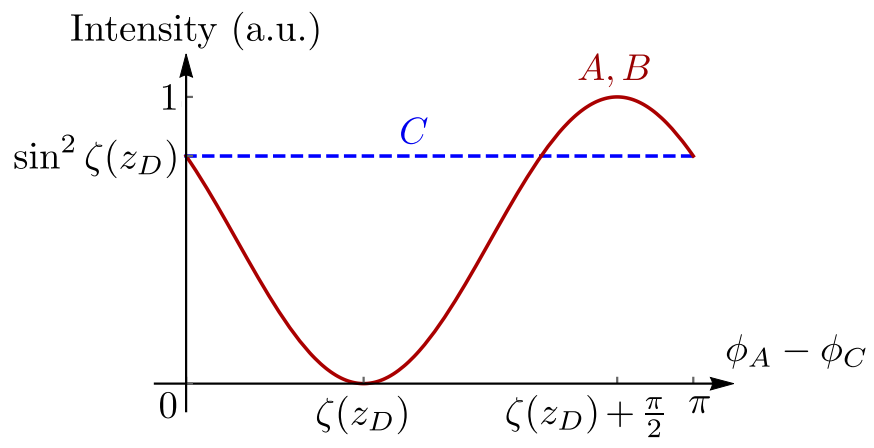

FIG. 3. The relative amplitudes of oscillations at frequencies $\omega_{A}$ and $\omega_{B}$ (both red, solid line) compared to that at $\omega_{C}$ (blue, dashed) in the power spectrum of (21) as a function of the static phase difference $\phi_{A}-\phi_{C}$. The value of $\zeta\left(z_{D}\right)$ was chosen arbitrarily to illustrate the point. (Color online)

from mirrors $E$ and $F$, which in turn agrees with the $1: 4$ ratios of the peaks in the power spectrum of the intensity. If path $B$ is brought completely out of phase with path $A$ and consequently with $C$, then $\left|\alpha_{0}\right|=1$, which explains the same factors in front of the three peaks $A, B$ and $C$ as in the above case as well as the disappearance of any peaks at $E$ and $F$ in the spectral analysis of the signal.

To complete our comparison with [2], we show the correspondence of (19) to the experimental results in which paths $F$ and $C$ are individually blocked. In the case of path $F$ or $C$ being blocked $e^{i \phi_{A, B}}$ or $e^{i \phi_{C}}$ must be replaced by zero, respectively, in (17). By examining (17) and (18) the consequences of these conditions may readily be seen. Blocking path $F$ results in a single peak at $C$ as the only non-vanishing coefficients in the output state are $\alpha_{C}(t)$ and crucially $\alpha_{0}$ showing that any light carrying information about the inner MZI has been prevented from reaching the detector. Blocking path $C$ results in the vanishing of $\alpha_{C}(t)$ and in $\left|\alpha_{0}\right|$ taking the value $\left|\exp \left(i \phi_{A}\right)+\exp \left(i \phi_{B}\right)\right|$. In the case when constructive interference is arranged in the inner MZI all peaks along the $A$ and $B$ paths show up as the carrier wave reaches the detector via these paths and so do all the perturbations caused by the mirrors along these paths. When destructive interference is arranged, however, $\alpha_{0}$ vanishes and - with the carrier wave absent - so does the entire signal.

\section{TUNABILITY OF THE PEAK HEIGHTS}

From (12), we can see that if the relative phase of the Gaussian and Hermite-Gaussian components could in principle be modified independently, the differential signal could be artificially strengthened or damped. For example, if the phase of the $\hat{a}_{1}^{\dagger}|0\rangle$ component of (9) was modified by an extra amount of $\zeta\left(z_{D}\right)-\zeta\left(z_{M}\right)$, the differential signal would vanish. Similarly, if the relative phase of the Hermite-Gaussian component was modified by $\zeta\left(z_{D}\right)-\zeta\left(z_{M}\right)-\frac{\pi}{2}$, the expectation value of $\widehat{\Delta I}$ would become the limit value, as if the detector was placed at infinity. In the case of a single tilted or vibrating mirror this is hypothetical because the two components are phase-locked. However, although unexplored in [2], the experiment readily provides means of achieving exactly this. All one needs to do is break the condition of the paths being aligned in phase (or completely out of phase). This can be best illustrated with path $C$ unblocked, paths $A$ and $B$ with opposite phases, and allowing an extra phase shift by statically displacing the mirror $C$. (In [2] only the mirror $B$ was displaced to control relative phases of the paths.) In this setting, only the term with $\delta_{C}(t)$ stays phase-locked to the Gaussian but the relative phase of $A$ and $B$ paths can be freely controlled. The values of the coefficients in (16) can then be written as

$$
\begin{aligned}
\alpha_{0} & =e^{i \phi_{C}}, \\
\alpha_{M}(t) & =e^{i \phi_{M}} i \delta_{M}(t), \quad M \in\{A, B, C\}, \\
\alpha_{E}(t) & =\alpha_{F}(t)=0
\end{aligned}
$$

and the detector signal, by inserting the above values into (18), as

$$
\left\langle\psi_{\text {out }}(t)|\widehat{\Delta I}| \psi_{\text {out }}(t)\right\rangle=\frac{2 I_{0}}{9}\left(\sin \left(\phi_{C}-\phi_{A}+\zeta\left(z_{D}\right)\right)\left(\delta_{A}(t)-\delta_{B}(t)\right)+\sin \zeta\left(z_{D}\right) \delta_{C}(t)\right) .
$$

Thus the relative strengths of the peaks at $A$ and $B$ to that of the peak at $C$ can be tuned by simply moving the mirror $C$, as plotted in Fig. 3. In particular, by matching the phase difference of $\phi_{A}-\phi_{C}$ to the Gouy phase $\zeta\left(z_{D}\right)$ (more precisely, to the Gouy phase difference $\zeta\left(z_{D}\right)-\zeta\left(z_{A, B}\right)$ had the approximation $z_{A, B} \ll z_{R}$ been not taken) and keeping $\phi_{B}-\phi_{A}=\pi$ fixed, the peaks at $A$ and $B$ (or either of them individually for $z_{A} \neq z_{B}$ ) may disappear completely. It would be absurd, of course, to conclude that the presence of the photons in the inner interferometer, or one of its arms, depends on the phase of the mirror $C$, or that not only are the paths taken by photons disconnected, but also sensitive to non-local conditions. 


\section{CONCLUSIONS}

We have modelled the experiment presented in [2] using only waves propagating forward in time and the interference of the possible paths. This allowed us to attribute the disappearance of peaks $E$ and $F$ in transitioning from maximum to minimum possible interference towards mirror $F$ to simple interference of paths in the inner Mach-Zehnder interferometer as mirrors $E$ and $F$ do not distinguish between paths $A$ and $B$. We have also shown that the simultaneous disappearance of all three remaining peaks when blocking path $C$ while maintaining destructive interference between the other two paths follows from the need for the perturbed wave $\hat{a}_{1}^{\dagger}|0\rangle$ to mix with the unperturbed carrier wave $\hat{a}_{0}^{\dagger}|0\rangle$. The necessary presence of $\hat{a}_{0}^{\dagger}|0\rangle$ for a non-vanishing signal means that interference between all the unblocked paths is a crucial part of the weak which-way measurement.

As a novel contribution to the discussion of this experimental setup we found the crucial dependence of the relative heights of the peaks in [2] on the three phases
$\phi_{A, B, C}$ associated with the three path lengths. In particular we have shown that some of these may be made to vanish by tuning the three path lengths. This shows by explicit construction that interpreting the lack of trace of a given mirror in the signal to mean that the photon has not interacted with that mirror is erroneous. It is this type of reasoning that led to the disconnected photon paths in [2]. We have found a hitherto unexplored tuning of the interferometer in which, without blocking any of the paths, the trace of only mirror $C$ is present in the output signal.

\section{ACKNOWLEDGMENTS}

The authors would like to thank Stephen M. Barnett for very insightful discussions and moral support. This research was funded by the EPSRC grant No. EP/I012451/1, "Challenges in Orbital Angular Momentum".
[1] L. Vaidman, "Past of a quantum particle," Phys. Rev. A 87, 052104 (2013).

[2] A. Danan, D. Farfurnik, S. Bar-Ad, and L. Vaidman, "Asking photons where they have been," Phys. Rev. Lett. 111, 240402 (2013).

[3] Z.-H. Li, M. Al-Amri, and M. S. Zubairy, "Comment on "Past of a quantum particle"," Phys. Rev. A 88, 046102 (2013).

[4] L. Vaidman, "Reply to "Comment on 'Past of a quantum particle""," Phys. Rev. A 88, 046103 (2013).

[5] H. Salih, "Comment on "Asking photons where they have been"," arXiv:1401.4888v2 (2014).

[6] P. L. Saldanha, "Interpreting a nested Mach-Zehnder interferometer with classical optics," Phys. Rev. A 89, 033825 (2014).

[7] A. Danan, D. Farfurnik, S. Bar-Ad, and L. Vaidman, "Reply to "Comment on 'Asking photons where have they been'"," arXiv:1401.5420v2 (2014).

[8] J.-H. Huang, L.-Y. Hu, X.-X. Xu, C.-J. Liu, Q. Guo, H.L. Zhang, and S.-Y. Zhu, "Comments on "Asking pho- tons where they have been"," arXiv:1402.4581 (2014).

[9] B. E. Y. Svensson, "Comments to "Asking photons where they have been" (A. Danan et al, Phys. Rev. Lett. 111, 240402 (2013))," arXiv:1402.4315v2 (2014).

[10] M. Wieśniak, "Criticism of "Asking photons where they have been"," arXiv:1407.1739 (2014).

[11] K. Bartkiewicz, A. Černoch, D. Javůrek, K. Lemr, J. Soubusta, and J. Svozilík, "One-state vector formalism for the evolution of a quantum state through nested Mach-Zehnder interferometers," Phys. Rev. A 91, 012103 (2015).

[12] Y. Aharonov, P. G. Bergmann, and J. L. Lebowitz, "Time symmetry in the quantum process of measurement," Phys. Rev. 134, B1410-B1416 (1964).

[13] Supplement I to [2].

[14] M. A. Bandres and M. Guizar-Sicairos, "Paraxial group," Optics Letters 34, 13-15 (2009).

[15] K.J. Blow, R. Loudon, S. J. D. Phoenix, and T. J. Shepherd, "Continuum fields in quantum optics," Physical Review A 42, 4102 (1990). 